[9] O. Deutschmann, Interaction between Transport and Chemistry in Catalytic Reactors, Habilitationsschrift, Universität Heidelberg 2001.

[10] R. J. Gorte, L. D. Schmidt, J. L. Gland, Surface Sci. 1981, 109 (2), 367.

[11] J. A. Serri, J. C. Tully, M. J. Cardillo, J. Chem. Phys. 1983, 79 (3), 1530

[12] E. I. Altman, R. J. Gorte, J. Phys. Chem. 1989, 93 (5), 1993.

[13] M. A. Morris, M. Bowker, D. A. King, C. H. Bamford, Comprehensive Chemical Kinetics - Simple Processes at the Gas-Solid Interface, Vol. 19, Elsevier, Amsterdam 1984

[14] M. E. Bartram, R. G. Windham, B. E. Koel, Surface Sci. 1987, 184 (1-2), 57.

[15] M. E. Bartram, R. G. Windham, B. E. Koel, Langmuir 1988, 4 (2), 240.

[16] D. T. Wickham, B. A. Banse, B. E. Koel, Surface Sci. 1989, 223 (1-2), 82.

[17] D. Chatterjee, Detaillierte Modellierung von Abgaskatalysatoren, Dissertation, Universität Heidelberg 2001

[18] Y. Y. Yeo, L. Vattuone, D. A. King, J. Chem. Phys. 1997, 106 (1), 392.

[19] L. Olsson, H. Persson, E. Fridell, M. Skoglundh, B. Andersson, J. Phys. Chem. B 2001, 105 (29), 6895.

[20] V. P. Zhdanov, B. Kasemo, Surface Sci. 1998, 415 (3), 403

[21] M. Crocoll, S. Kureti, W. Weisweiler, J. Catal., eingereicht.

\section{Fließeigenschaften feinstdisperser, gesättigter Haufwerke während ihrer Kompression im Zentrifugalfeld}

ATEŞ ERK *, HARALD ANLAUF UND WERNER STAHL

\section{Problemstellung}

Die Fest/Flüssig-Trennung von Suspensionen feinster Partikeln (Partikeldurchmesser um $1 \mu \mathrm{m}$ und darunter) mittels Filtrations- und Sedimentationsverfahren ist eine verfahrenstechnische Aufgabe von großer wirtschaftlicher Bedeutung. Beispiele hierfür sind die Herstellung von Pigmenten

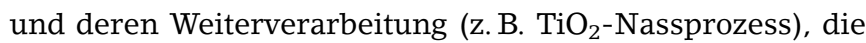
Verarbeitung oxidkeramischer Grundstoffe (z. B. $\mathrm{Al}_{2} \mathrm{O}_{3}$ ), die Herstellung von Beschichtungs- und Veredelungsmaterialien für die Papierindustrie (z. B. Kaolin, $\mathrm{CaCO}_{3}$ ) oder auch die Behandlung industrieller und kommunaler Abwässer.

Die verfahrenstechnische Funktion des Trennprozesses und angrenzender Grundoperationen wird bei

* Dipl.-Ing. A.ERK (ates.erk@mvm.uka.de) Dr.-Ing. H. ANLAUF, Prof. Dr.-Ing. W. STAHL, Institut für Mechanische Verfahrenstechnik und Mechanik, Universität Karlsruhe (TH) Kaiserstr. 12, D-76128 Karlsruhe, Germany. derart oberflächenreichen Systemen meist stark durch die rheologischen Eigenschaften des verarbeiteten Produkts beeinflusst, was in der Literatur vielfach bestätigt wird [1 6]. Einerseits muss das Fließverhalten des ausgetragenen Produkts bestimmten Anforderungen genügen, um die Funktion von sich an die Fest/Flüssig-Trennung anschließenden Apparaten (z. B. Fördereinrichtungen, Mischer, Trockner) zu gewährleisten. Andererseits hängt die Funktion vieler Trennapparate selbst maßgeblich von den Fließeigenschaften des Fest/Flüssig-Systems ab. Hierbei spielen vor allem der Austrag der feststoffreichen Phase (Sediment, Filterkuchen) sowie, im Fall kontinuierlicher Prozesse, deren Förderung innerhalb des Trennapparates (Drehmomententstehung, Förderbarkeit) eine große Rolle.

Hinsichtlich der rheologischen Eigenschaften sind aus verfahrenstechnischer Sicht Materialwiderstände gegen plastische Verformung, d.h. Fließgrenzen, von übergeordneter Bedeutung [3, 7]. Haupteinflussparameter auf Fließgrenze und Kompressionsverhalten gesättigter, feinstdisperser Haufwerke sind einerseits Konzentration und disperse Eigenschaften der festen Phase (Partikelgröße, Partikelgrößenverteilung, spez. Oberfläche, Partikelform). Andererseits spielen die Grenzflächeneigenschaften der suspendierten Partikeln im Zusammenspiel mit dem umgebenden Medium und die daraus resultierende Größe und Reichweite der interpartikulären Wechselwirkungen eine erhebliche Rolle. Diese bestimmen den Agglomerationszustand sowie die Mikrostruktur des Systems. Die interpartikulären Kräfte können vereinfacht in attraktive van der Waals- und in der Regel repulsive elektrostatische Kräfte unterteilt und anhand des sog. Zeta-Potentials charakterisiert werden $[8,9]$. Bei hohen Beträgen des Zeta-Potentials dominieren abstoßende Wechselwirkungen, während bei einem Zeta-Potential von Null (am sog. isoelektrischen Punkt, IEP) maximale Anziehung vorliegt.

Sobald sich in einem Fest/Flüssig-System Partikeln aufeinander abstützen, besteht zudem eine Abhängigkeit der Fließgrenze vom wirksamen Feststoffgerüstdruck. Dies muss beachtet werden, wenn die Fließgrenze eines während des Trennprozesses unter Belastung stehenden Haufwerks erfasst werden soll. Die Gerüstdruckabhängigkeit der Fließgrenze wird anhand sog. Fließorte im Schubspannungs-Normalspannungs-Diagramm beschrieben.

Feinstpartikuläre Haufwerke sind meist hochkompressibel, d.h. der Feststoffgerüstdruck beeinflusst neben der Fließgrenze auch in starkem Maße die Feststoffkonzentration. Er entsteht durch Flüssigkeitsreibungskräfte (Filtration), Massenkräfte (z. B. Zentrifugation), äußere Kompressionskräfte (Pressfiltration) oder durch Kombinationen davon. Die genannten Kräfte sind entlang der Haufwerkshöhe in der Regel nicht konstant, weshalb sich ein Gerüstdruckgradient und somit auch ein Feststoffkonzentrationsgradient im Haufwerk ausbilden. Dies führt dazu, dass in vielen Fest/Flüssig-Trennapparaten eine zeitliche und örtliche Verteilung der Fließeigenschaften vorliegt, die häufig die verfahrenstechnischen Vorgänge im Apparat und somit dessen Funktion dominiert.

In der vorliegenden Arbeit werden Ergebnisse bezüglich der Fließgrenze von Sedimenten im Zentrifugal- 
feld präsentiert. Da diese messtechnisch nicht direkt zugänglich sind, werden die Fließgrenzen aus einer Kombination von Kompressions- und Scherexperimenten ermittelt. Die erhaltenen Daten geben Aufschluss über das Materialverhalten im Trenngerät und sind beispielsweise für die Auslegung von Dekantierzentrifugen und Tellerseparatoren relevant.

\section{Methoden}

Als Versuchsprodukte wurden wässrige Suspensionen feinstdisperser Kaolin- und Kalksteinpulver verwendet. Die Eigenschaften der verwendeten Stoffsysteme sind in Tab. 1 dargestellt.

Tabelle 1.

Eigenschaften der verwendeten Versuchsprodukte.

\begin{tabular}{lll}
\hline Fluid & demin. $\mathrm{H}_{2} \mathrm{O}$ & \\
Feststoff & Kaolin & Kalkstein \\
Bezeichnung & H1 Spezial & OmyaCarb Extra CL \\
Hersteller & Dorfner & Omya \\
$x_{50,3} / \mu \mathrm{m}$ & 0,9 & 1,2 \\
$S_{\mathrm{V}} / 10^{6} \cdot \mathrm{m}^{-1}$ & 5,9 & 5,7 \\
$\rho_{\mathrm{s}} / \mathrm{kg} \cdot \mathrm{m}^{-} 3$ & 2600 & 2720 \\
\hline
\end{tabular}

Zur Haufwerksbildung wurde eine Becherzentrifuge des Typs BHG Hermle ZK630 mit schwenkbar gelagerten Gehängebechern eingesetzt (max. 9000 g). Dabei wurden die Suspensionen bei definierter Drehzahl bis zum Erreichen des Kompressionsgleichgewichts der entstandenen Sedimente zentrifugiert. Anschließend wurden die Sedimente mittels eines Ausschubkolbens aus den Zentrifugenbechern entnommen und dabei in $2 \mathrm{~mm}$ dicke Scheiben geschnitten, deren Feststoffkonzentration jeweils gravimetrisch ermittelt wurde. Unter Vernachlässigung von Rückdehnungseffekten ist auf diese Weise bei bekannter Dichte des Feststoffs und der Flüssigkeit die Bestimmung der Feststoffkonzentration entlang der Sedimenthöhe möglich (Gl. (1)). Ferner lässt sich der Feststoffgerüstdruck während der Zentrifugation in der Mitte der jeweiligen Sedimentschicht $i$ nach Gl. (2) berechnen [10]. Dabei sind die Schichten oberhalb der Schicht $i$ mit $h$ indiziert ${ }^{1)}$.

$c_{\mathrm{V}}=\frac{V_{\mathrm{s}}}{V_{\mathrm{s}}+V_{1}}=\frac{m_{\mathrm{s}}}{m_{\mathrm{s}}+\frac{\rho_{\mathrm{S}}}{\rho_{1}} m_{1}}$

$p_{\mathrm{s}, \mathrm{i}}=\omega^{2} \frac{\rho_{\mathrm{s}}-\rho_{1}}{\rho_{\mathrm{s}}} \frac{4}{\pi D_{\mathrm{B}}^{2}} \sum_{\mathrm{h}=1}^{\mathrm{i}-1} R_{\mathrm{h}} \Delta m_{\mathrm{s}, \mathrm{h}}+\frac{R_{\mathrm{i}} \Delta m_{\mathrm{s}, \mathrm{i}}}{2}$

Diese Daten bilden die Basis für die anschließende Berechnung der während der Zentrifugation vorliegenden Fließgrenzprofile. Neben den Daten bezüglich der Sedimentverdichtung und -belastung werden hierzu rheologische Materialfunktionen benötigt, d. h. die Fließgrenze

1) Eine Zusammenstellung der Formelzeichen befindet sich am Schluss des Beitrags. als Funktion der Feststoffkonzentration und des Feststoffgerüstdruckes (Fließort).

Die Normalspannungsabhängigkeit der Fließgrenze wurde mittels einer filtrierend ausgeführten Hochdruck-Jenike-Scherzelle (offenes System) bestimmt. Diese besteht aus zwei übereinanderliegenden Ringen, die gegeneinander verschiebbar sind. Die Stirnseiten der so gebildeten Scherzelle, von denen eine beweglich ausgeführt ist, sind perforiert und mit Filtermedien belegt (s. Skizze in Abb. 3). Die gesamte Anordnung ist in eine Universalprüfmaschine integriert, welche die Vorgabe der Schergeschwindigkeit sowie Messung von Scherkraft-ScherwegVerläufen beim Verschieben der Ringe gestattet. Die Verwendung eines Pneumatikzylinders und eines Presskolbens ermöglicht dabei die Belastung der Probe mit vorgebbaren Normalspannungen von bis zu 1,5 $\mathrm{MPa}$.

Die Vorgehensweise zur Ermittlung von Fließorten entsprach der aus der Schüttgutmechanik bekannten Methode [7]. Zunächst wurde eine im Konsolidierungsgleichgewicht befindliche Probe unter Belastung bis zum Erreichen einer konstanten Scherspannung angeschert, um eine homogene Struktur zu gewährleisten. Anschließend erfolgten Abschervorgänge bei reduzierten Normalspannungen. Die Versuche wurden für unterschiedliche Anscherspannungen und somit Feststoffkonzentrationen durchgeführt. Als Resultat wurde eine Fließortschar erhalten, welche die Abhängigkeit der Fließgrenze von der Fest- 
stoffkonzentration und der Normalspannung, die mit dem Feststoffgerüstdruck gleichzusetzen ist, beschreibt.

\section{Ergebnisse}

Zunächst wurden Kompressionsversuche in der Becherzentrifuge bei unterschiedlichen Zentrifugalbeschleunigungen durchgeführt und die jeweiligen Feststoffkonzentrationsund Gerüstdruckprofile bestimmt. Abb. 1 zeigt exemplarisch die Feststoffkonzentration entlang der Höhe eines Kaolin- und eines Kalksteinsediments, die bei 2640 g (im Folgenden genannte Zentrifugalbeschleunigungen bzw. C-Werte beziehen sich auf den maximalen Radius) bis zum Erreichen des Gleichgewichtszustands zentrifugiert wurden. Es ist erwartungsgemäß zu erkennen, dass zum Sedimentboden hin die Feststoffkonzentration ansteigt und der Konzentrationsgradient abnimmt. Das Kalksteinsediment erreicht dabei eine höhere Feststoffkonzentration. Der experimentell bestimmte Verlauf des Feststoffgerüstdrucks entlang der Sedimenthöhe ist für das Kaolinhaufwerk in Abb. 2 dargestellt. Das Gerüstdruckprofil ist annähernd linear, d.h. sowohl der Anstieg der Feststoffkonzentration als auch der Zentrifugalbeschleunigung zum Becherboden hin wirken sich im betrachteten Fall nur geringfügig auf den

Abbildung 1.

Feststoffkonzentrationsprofile eines Kaolin- und eines Kalksteinsediments, bei $2640 \mathrm{~g}$ bis zum Erreichen des Gleichgewichtszustands (GG) zentrifugiert $(x=0$ : Becherboden).

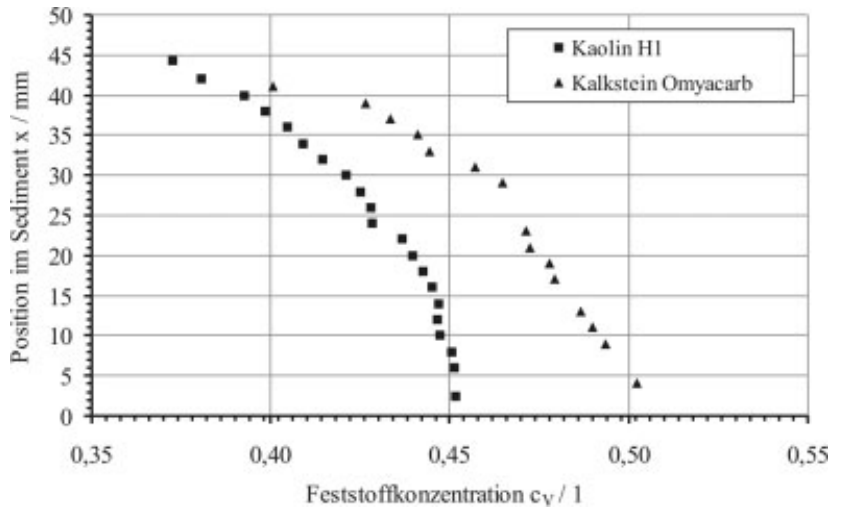

Abbildung 2.

Feststoffgerüstdruck entlang der Höhe eines Kaolinsediments (2640 g, GG).

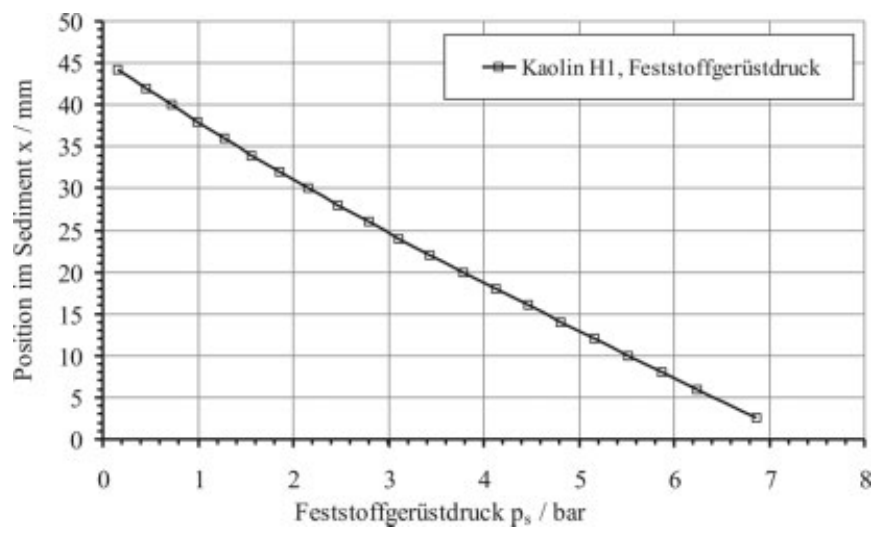

Gerüstdruck aus. Am Becherboden wird ein Gerüstdruck von über $700 \mathrm{kPa}$ (7 bar) erreicht.

Abb. 3 zeigt Fließorte für Kaolinhaufwerke unterschiedlicher Feststoffkonzentrationen im Bereich zwischen $c_{\mathrm{v}}=0,38-0,45$ (s. Abb. 1). Die zugehörigen Anscherspannungen und Feststoffkonzentrationen sind im Diagramm eingetragen. Bei allen Scherversuchen wurde mit einer Schergeschwindigkeit von $0,5 \mathrm{~mm} / \mathrm{min}$ gearbeitet. Zusammen mit den Daten aus den Abbn. 1 und 2 lässt sich die Fließgrenze entlang der Sedimenthöhe in belastetem Zustand darstellen (s. Abb. 4). Hierzu wird vereinfachend am für die jeweilige lokale Feststoffkonzentration gültigen Fließort die Schubspannung beim entsprechenden Feststoffgerüstdruck, der mit der Normalspannung gleichgesetzt wird, abgelesen.

Abb. 4 zeigt dabei neben den exakten Werten auch solche Fließgrenzverläufe, die aus einer Approximation der Fließorte mittels einer einzigen Geraden der Steigung $\tan \varphi$ (gestrichelte Linie in Abb. 3) abgeleitet wurden. Diese Vereinfachung lässt sowohl den Einfluss der Feststoffkonzentration als auch die Krümmung der Fließorte unbeachtet, ergibt jedoch, wie in Abb. 4 zu erkennen, im betrachteten Fall lediglich geringe Abweichungen vom exakten Verlauf. Das Verfahren, das in ähnlicher Weise bereits von WEBER [3] angewandt wurde, ist somit für praxisnahe Aussagen zulässig. Analog zu Kaolin wurde mit Kalksteinsedimenten verfahren. Dabei ergibt sich im Vergleich, dass Kalksteinsedimente bei identischen Zentrifugations-

Abbildung 3.

Fließorte gesättigter Kaolinhaufwerke bei unterschiedlichen Anscherspannungen bzw. Feststoffkonzentrationen sowie lineare Approximation (gestrichelte Gerade).

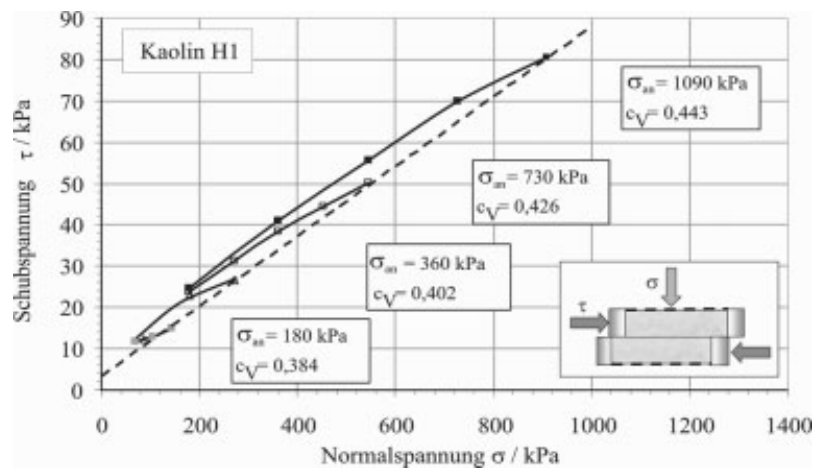

Abbildung 4.

Fließgrenzen während der Zentrifugation entlang der Höhe eines Kaolin- und eines Kalksteinsediments (2640 g, GG).

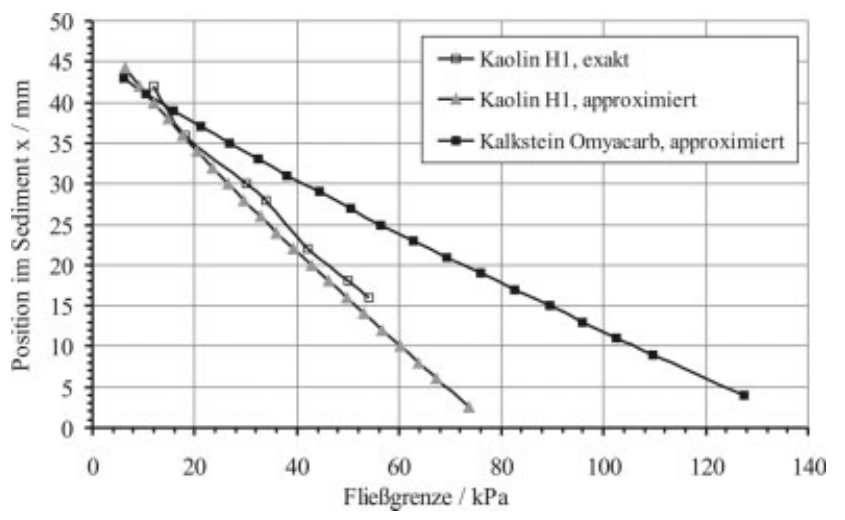


bedingungen wesentlich höhere Festigkeiten aufweisen als Kaolinsedimente, was auf einen höheren Reibwinkel $\varphi$ zurückzuführen ist.

Abb. 5 zeigt den Einfluss des C-Wertes (Vielfaches der Erdbeschleunigung) auf das Fließgrenzprofil von Kaolinsedimenten (Gleichgewichtszustand). Dabei ist ersichtlich, dass die maximale Fließgrenze am Außenradius dem C-Wert etwa proportional ist. Überträgt man dies auf Tellerseparatoren mit einem C-Wert um 10000 , so wird ersichtlich, dass bei zu langer Verweilzeit, d.h. Kompression bis in die Nähe des Gleichgewichts, mineralische Sedimente derart verfestigt werden, dass das Austragssystem der Zentrifuge blockiert werden kann. Hierbei sind insbesondere Produkte mit hoher Dichtedifferenz $\Delta \rho$ zwischen Feststoff und Flüssigkeit und einem hohen Reibwinkel $\varphi$ kritisch. Im Fall von Dekantierzentrifugen ist bei solchen Stoffsystemen mit einer hohen Drehmomententstehung an der Transportschnecke zu rechnen. Wässrige biologische Systeme verhalten sich hingegen weniger kritisch, da sie in der Regel nicht die genannten Eigenschaften aufweisen, d.h. Dichtedifferenz und Reibwinkel sind vergleichsweise gering. Entsprechend verursachen sie in selbstentleerenden Tellerseparatoren weniger Probleme beim Austrag. In Dekantierzentrifugen können zu niedrige Fließgrenzen jedoch zu Förderschwierigkeiten am Konus führen.

Abbildung 5.

Fließgrenzen während der Zentrifugation entlang der Höhe eines Kaolinsediments bei unterschiedlichen C-Werten (GG).

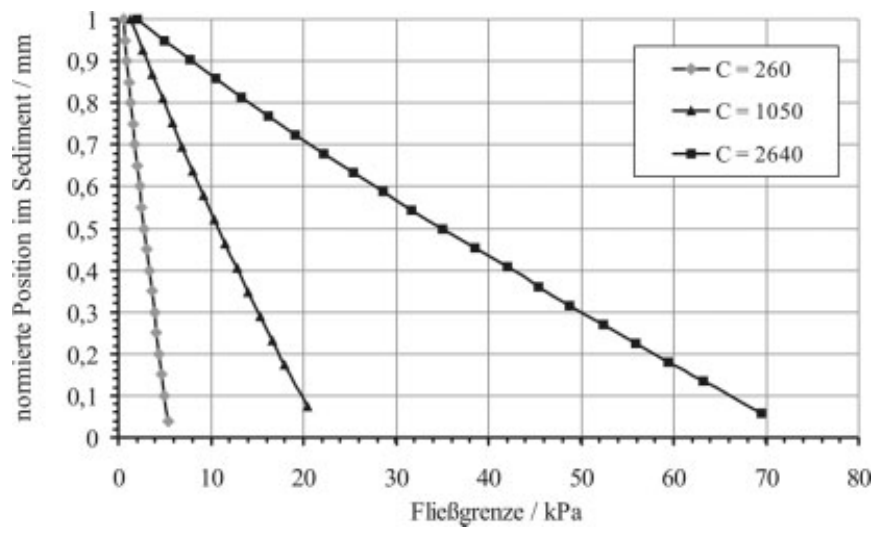

Um den Einfluss unterschiedlicher interpartikulärer Wechselwirkungen auf die Festigkeit unter Belastung stehender Sedimente zu untersuchen, wurden die Kompressions- und Scherexperimente bei variierten $\mathrm{pH}$-Werten durchgeführt. Dabei wurden der IEP $(\varsigma=0)$ sowie ein pH-Wert, bei dem ein betragsmäßig hohes Zeta-Potential vorliegt, für die Versuche ausgewählt (Kaolin: $\mathrm{pH}=10$, $\varsigma=-50 \mathrm{mV})$.

In Abb. 6 sind zwei Fließorte eines Kaolinhaufwerks bei annähernd gleicher Feststoffkonzentration, jedoch unterschiedlichem $\mathrm{pH}$-Wert dargestellt. Am IEP ist der Fließort zu höheren Schubspannungen hin verschoben. Die unterschiedlichen Enddrücke der Fließorte ergeben sich aus der Tatsache, dass die $\mathrm{pH}$-Verschiebung neben der Fließgrenze auch das Kompressionsverhalten beeinflusst. Bei anziehenden interpartikulären Wechselwirkungen am IEP entsteht eine festere Struktur, d. h. zum Erreichen der
Abbildung 6.

Fließorte gesättigter Kaolinhaufwerke bei unterschiedlichem pH-Wert bzw. Zeta-Potential und annähernd konstanter Feststoffkonzentration.

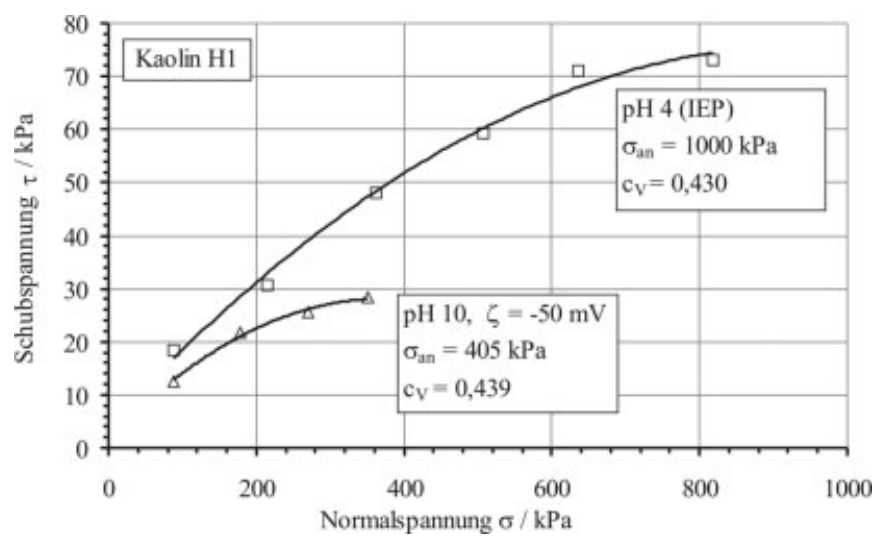

Abbildung 7.

Fließgrenzen während der Zentrifugation entlang der Höhe von Kaolinsedimenten bei identischen Zentrifugationsbedingungen $(2640 \mathrm{~g}, \mathrm{GG})$, jedoch unterschiedlichem pH-Wert bzw. Zeta-Potential.

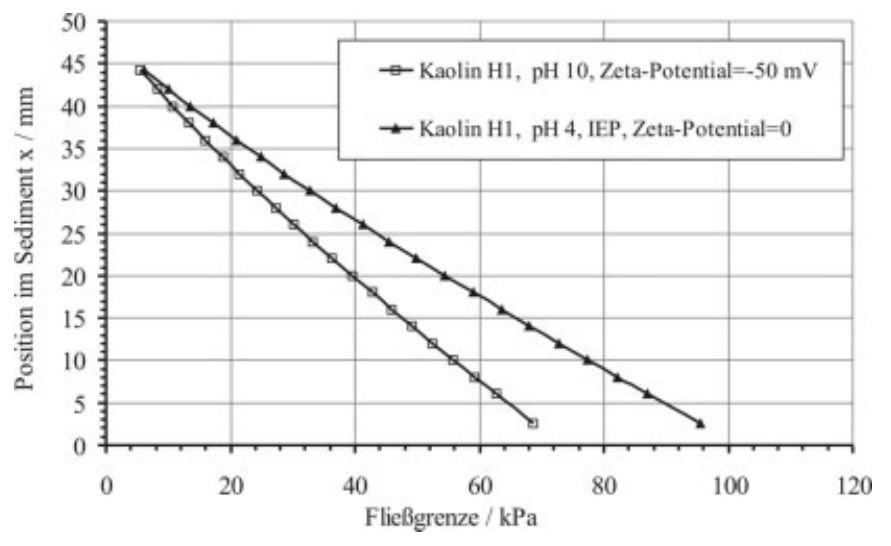

gleichen Feststoffkonzentration muss ein höherer Feststoffgerüstdruck aufgeprägt werden als bei abstoßenden interpartikulären Wechselwirkungen $(\mathrm{pH}=10)$.

Leitet man aus diesen Ergebnissen wiederum Fließgrenzprofile entlang der Sedimenthöhe ab, so ergeben sich die in Abb. 7 dargestellten Verläufe. Die pH-Verschiebung führt im Fall von Kaolin zu einem Anstieg der Fließgrenze am Außenradius um ca. 40 \%. Es ist anzumerken, dass der Einfluss interpartikulärer Wechselwirkungen auf das Fließ- und Kompressionsverhalten mit abnehmender Partikelgröße stark anwächst. So ist beispielsweise im Falle von Pigmenten (z. B. $\mathrm{TiO}_{2}$ ) oder Keramikpulvern (z.B. $\mathrm{Al}_{2} \mathrm{O}_{3}$ ) mit Partikelgrößen um $300-400 \mathrm{~nm}$ ein ausgeprägterer Effekt zu erwarten.

\section{Schlussfolgerung}

In der vorliegenden Arbeit wurden Fließgrenzprofile zentrifugierter Sedimente unter Prozessbedingungen bestimmt, d. h. unter einer der Zentrifugalkraft entsprechenden Belastung. Die Ergebnisse wurden durch eine Kombination von Kompressions- und Scherversuchen ermittelt und beziehen sich auf Gleichgewichtszustände. Es wurde gezeigt, dass die 
Fließgrenze zum Sedimentboden hin aufgrund des sich erhöhenden Feststoffgerüstdruckes und der ansteigenden Feststoffkonzentration stark zunimmt. Der C-Wert hat einen maßgeblichen Einfluss auf das Fließgrenzprofil, da er zusammen mit der Dichtedifferenz zwischen Feststoff und Flüssigkeit der Haupteinflussparameter auf den Feststoffgerüstdruck ist. Ferner besteht ein erheblicher Einfluss des Produktreibwinkels auf die Sedimentfließgrenze.

Eine Steigerung der anziehenden interpartikulären Wechselwirkungen durch Änderung des pH-Wertes wirkte sich makroskopisch in Form einer Erhöhung der Sediment-Fließgrenze aus. Die Änderung der Sedimentfestigkeit (Verfestigung oder Verflüssigung) durch Variation des pH-Wertes bietet, im Gegensatz zum Einsatz polymerer Flockungshilfsmittel, eine annähernd reversible und fremdstofffreie Möglichkeit, das rheologische Materialverhalten an die jeweiligen Anforderungen des Fest/Flüssig-Trennprozesses bzw. -apparates anzupassen. Diese Aussage gilt nicht nur für Zentrifugen, sondern lässt sich ebenso auf Doppelbandpressen, Querstromfilter sowie Misch- und Fördereinrichtungen ausweiten.

\section{Ausblick}

Neben dem Gleichgewicht ist für die Auslegung industrieller Fest/Flüssig-Trennprozesse die Kinetik der Verdichtung und Verfestigung feinstpartikulärer Haufwerke von großer Wichtigkeit, da die Verweilzeiten aus wirtschaftlichen Gründen möglichst kurz gehalten werden müssen. Angesichts bereits bestehender Probleme in der Praxis sowie der Notwendigkeit, zunehmend feinere Partikelsysteme zu verarbeiten und zu handhaben, besteht der Bedarf, die Grundlagen für eine physikalisch begründete Beschreibung des Einflusses rheologischer Eigenschaften auf Fest/FlüssigTrennprozesse zu schaffen.

Die Kompressionskinetik gesättigter Fest/FlüssigSysteme kann mittels eines differentiellen Ansatzes sowie der numerischen Lösung der notwendigen Gleichungen modellhaft beschrieben werden (Filtration und Zentrifugation) [11]. Am Institut für MVM der Universität Karlsruhe (TH) wurde hierzu ein Simulationsprogramm entwickelt $[5,12]$ das die zeitaufgelöste Berechnung lokaler Feststoffkonzentrationen und Gerüstdrücke ermöglicht. Durch Kopplung dieses Ansatzes mit rheologischen Materialfunktionen soll die orts- und zeitaufgelöste Vorausberechnung der Fließgrenze in einem sich verdichtenden Haufwerk realisiert werden.

Die Untersuchungen sollen ferner auf Systeme mit Partikelgrößen deutlich unter $1 \mu \mathrm{m}$ ausgeweitet werden. Besondere Beachtung verdient dabei der Einfluss der physiko-chemischen Bedingungen auf die Fließ- und Kompressionseigenschaften, der im Falle feinstdisperser Systeme von entscheidender Bedeutung ist.

Eingegangen am 29. Juli 2004 [CIT 7025]

\section{Formelzeichen}

\begin{tabular}{|c|c|c|}
\hline $\mathrm{C}$ & {$[-]$} & Vielfaches der Erdbeschleunigun \\
\hline$c_{\mathrm{V}}$ & {$[-]$} & $\begin{array}{l}\text { Volumenkonzentration des Fest- } \\
\text { stoffs }\end{array}$ \\
\hline$D_{\mathrm{B}}$ & {$[\mathrm{m}]$} & $\begin{array}{l}\text { Innendurchmesser des Zentrifu- } \\
\text { genbechers }\end{array}$ \\
\hline g & {$\left[\mathrm{m} \mathrm{s}^{-2}\right]$} & Erdbeschleunigung \\
\hline$h$ & {$[-]$} & Laufvariable \\
\hline$i$ & {$[-]$} & Laufvariable \\
\hline$m_{\mathrm{s}}$ & {$[\mathrm{kg}]$} & Masse des Feststoffs \\
\hline$m_{1}$ & {$[\mathrm{~kg}]$} & Masse der Flüssigkeit \\
\hline$\Delta m_{\mathrm{s}, \mathrm{i}}$ & {$[\mathrm{kg}]$} & $\begin{array}{l}\text { Feststoffmasse in der Sediment- } \\
\text { schicht } i\end{array}$ \\
\hline$\Delta m_{\mathrm{s}, \mathrm{h}}$ & {$[\mathrm{kg}]$} & $\begin{array}{l}\text { Feststoffmasse in der Sediment- } \\
\text { schicht } h\end{array}$ \\
\hline$p_{\mathrm{s}}$ & {$[\mathrm{Pa}]$} & Feststoffgerüstdruck \\
\hline$p_{\mathrm{s}, \mathrm{i}}$ & {$[\mathrm{Pa}]$} & $\begin{array}{l}\text { Feststoffgerüstdruck in der Sedi- } \\
\text { mentschicht } i\end{array}$ \\
\hline$R$ & {$[\mathrm{~m}]$} & Zentrifugationsradius \\
\hline$R_{\mathrm{h}}$ & {$[\mathrm{m}]$} & $\begin{array}{l}\text { Zentrifugationsradius der Sedi- } \\
\text { mentschicht } h\end{array}$ \\
\hline$R_{\mathrm{i}}$ & {$[\mathrm{m}]$} & $\begin{array}{l}\text { Zentrifugationsradius der Sedi- } \\
\text { mentschicht } i\end{array}$ \\
\hline$S_{\mathrm{V}}$ & {$\left[\mathrm{m}^{-1}\right]$} & volumenspezifische Oberfläche \\
\hline$V_{\mathrm{s}}$ & {$\left[\mathrm{m}^{3}\right]$} & Volumen des Feststoffs \\
\hline$V_{1}$ & {$\left[\mathrm{~m}^{3}\right]$} & Volumen der Flüssigkeit \\
\hline$v_{\mathrm{s}}$ & {$\left[\mathrm{m} \mathrm{s}^{-1}\right]$} & Schergeschwindigkeit \\
\hline$x$ & {$[\mathrm{~m}]$} & Ortskoordinate \\
\hline$x_{50,3}$ & {$[\mathrm{~m}]$} & Medianwert der $Q_{3}$-Verteilung \\
\hline
\end{tabular}

griechische Buchstaben

$\begin{array}{lll}\varphi & {[-]} & \text { Reibwinkel } \\ \rho_{\mathrm{s}} & {\left[\mathrm{kg} \mathrm{m}^{-3}\right]} & \text { Feststoffdichte } \\ \rho_{\mathrm{l}} & {\left[\mathrm{kg} \mathrm{m}^{-3}\right]} & \text { Flüssigkeitsdichte } \\ \sigma & {[\mathrm{Pa}]} & \text { Normalspannung } \\ \tau & {[\mathrm{Pa}]} & \text { Schubspannung } \\ \tau_{\mathrm{f}} & {[\mathrm{Pa}]} & \text { Fließgrenze } \\ \omega & {\left[\mathrm{s}^{-1}\right]} & \text { Winkelgeschwindigkeit } \\ \varsigma & {[\mathrm{V}]} & \text { Zeta-Potential }\end{array}$

A b kürzungen
IEP $\quad$ isoelektrischer Punkt
GG $\quad$ Gleichgewichtszustand

\section{Literatur}

[1] H. Riemenschneider, Dissertation, Universität Stuttgart 1983.

[2] A. Karolis, Dissertation, Universität Karlsruhe (TH) 1986.

[3] J. Weber, Dissertation, Universität HamburgHarburg 1992.

[4] B. Reichmann, Dissertation, Otto-von-GuerickeUniversität Magdeburg 1999.

[5] J. Heuser, Dissertation, Universität Karlsruhe (TH) 2003.

[6] A. Erk, W. Stahl, Proc. FiltechEurope, Düsseldorf 2003. 
[7] J. Schwedes et al., Hochschulkurs Lagern und Fließen von Schüttgütern, Braunschweig 2004.

[8] P. J. Scales, P. C. Kapur, S. B. Johnson, T. W. Healy, AIChE J. 1998, 44, 538.

[9] G. Lagaly, O. Schulz, R. Zimehl, Dispersionen und Emulsionen, Steinkopff-Verlag, Darmstadt 1997.

[10] H. Reinach, Dissertation, Universität Karlsruhe (TH) 1992.

[11] P. Garrido, F. Concha, R. Bürger, Int. J. Miner. Process. 2003, 72, 57.

[12] C. M. Alles, Dissertation, Universität Karlsruhe (TH) 2000.

\section{Deaktivierung von SCR-DeNO ${ }_{x^{-}}$ Katalysatoren durch Phosphor- verbindungen*}

JENS BECK * *, JÜRGEN BRANDENSTEIN, SVEN UNTERBERGER UND KLAUS R. G. HEIN

\section{Problemstellung}

Die Mitverbrennung von phosphorreichen Sekundärbrennstoffen wie Klärschlamm oder auch Tiermehl kann zu starken Deaktivierungsraten von den in Kohlekraftwerken installierten SCR-Entstickungskatalysatoren führen. Daraus folgen erhebliche Mehraufwendungen der Kraftwerksbetreiber für die Regenerierung oder auch den vorzeitigen Austausch kompletter Katalysatorlagen.

In vorhergehenden Arbeiten wurde ein Zusammenhang zwischen Aktivitätsverlust und Phosphorkonzentration in der Katalysator-Randschicht hergestellt. In Laborversuchen sowie in Verbrennungsversuchen im Technikumsmaßstab wurde der Einfluss von Phosphat im Rauchgas sowie im Brennstoff auf die Aktivität des Katalysators untersucht. Die Laboruntersuchungen zeigten eine starke Abnahme der Katalysatoraktivität bei Zugabe von Phosphat in einem synthetischen Rauchgas. Dies wurde von Verbrennungsversuchen mit realem Rauchgas im Wesentlichen bestätigt, wobei unterschiedliche Phosphate sich verschieden stark auf die Aktivitätsabnahme auswirkten.

Die deaktivierende Wirkung von Phosphorverbindungen auf Katalysatoren wurde bereits vor 25 Jahren an Edelmetallkatalysatoren beschrieben [1]. Insbesondere die in Treibstoffen und Schmiermitteln enthaltenen Phosphorverbindungen führen bei edelmetallhaltigen Kraftfahrzeug-

* Vortrag anlässlich der Sitzung des GVCFachausschusses "Gasreinigung", 16./17. Februar 2004 in Karlsruhe.

* * Dipl.-Min. J. BRANDENSteIn, E.On EngiNEERING GMBH, Bergmannsglückstraße 41-43, D-45896 Gelsenkirchen, Germany; MEng. J.BECK (Korrespondenzautor, beck@ ivd.uni-stuttgart.de), Dipl.-Ing. S. UNTERBERGER, Prof. Dr. K. R.G. HEIN, Institut für Verfahrenstechnik und Dampfkesselwesen, Pfaffenwaldring 23, D-70569 Stuttgart, Germany. katalysatoren zur Bildung von Oberflächenschichten am Katalysatoreintritt [2].

Bedingt durch den zumeist sehr geringen Phosphorgehalt in Kohle wurde bisher in Kohlefeuerungen keine Deaktivierung durch Phosphor festgestellt. Erst beim Einsatz phosphorreicher Sekundärbrennstoffe, insbesondere von Klärschlamm und Tiermehl, wurde im Betrieb eine erhöhte Abnahme der Aktivität der SCR-DeNOx-Katalysatoren festgestellt.

ANDERLE [3] beschrieb das ternäre System $\mathrm{P}_{2} \mathrm{O}_{5}$ / $\mathrm{V}_{2} \mathrm{O}_{5} / \mathrm{TiO}_{2}$. Demzufolge findet eine Wechselwirkung zwischen Phosphor, Titandioxid und Vanadyl statt. Dabei werden amorphe V-P-O-Einheiten gebildet. Durch die gleichzeitige Erhöhung der Sauerstoff-Bindungsstärke ist die Sauerstoffmobilität herabgesetzt, und die Redoxeigenschaften werden verändert. Diese Veränderungen nehmen mit steigender P-Konzentration zu. Ab 3 Gew.-\% P auf dem Katalysator wurde die Bildung von mikrokristallinen $\mathrm{VOPO}_{4}$-Gruppen beobachtet.

BLANCO et. al. wiesen die deaktivierende Wirkung von Orthophosphorsäure $\mathrm{H}_{3} \mathrm{PO}_{4}$ auf $\mathrm{V}_{2} \mathrm{O}_{5}$-Katalysatoren im Labormaßstab nach [4]. Im Anschluss zeigten CHEN et. al. [5] die Deaktivierung von SCR-DeNOx-Katalysatoren durch $\mathrm{P}_{2} \mathrm{O}_{5}$ in Abhängigkeit von dem P/V-Verhältnis und der Temperatur. Weiterhin legten BUSCA et al. [6] die Interaktion von Phosphaten mit wesentlichen Bestandteilen von SCRDeNOx-Katalysatoren wie $\mathrm{MoO}_{3}, \mathrm{WO}_{3}$ und $\mathrm{V}_{2} \mathrm{O}_{5}$ nahe. Die Betriebserfahrungen aus der Mitverbrennung wiesen ebenfalls auf einen negativen Einfluss von P auf die Aktivität der DeNOx-Katalysatoren [7] hin.

Um einen Zusammenhang zwischen P-Beladung und Deaktivierung festzustellen, wurden Katalysatorproben aus der Klärschlamm- und Tiermehl-Mitverbrennung untersucht. Dabei wurde ein Verlust der katalytischen Aktivität, verbunden mit einer Phosphoranreicherung von zum Teil über 4 Gew.-\%, festgestellt. Aus den Ergebnissen wurden analog zu den Untersuchungen von BLANCO und CHEN mögliche Deaktivierungsmechanismen abgeleitet, die zunächst von der Bildung von gasförmigem Phosphoroxid $\mathrm{P}_{2} \mathrm{O}_{5}$ sowie Orthophosphat $\mathrm{PO}_{4}{ }^{3-}$ während der Verbrennung ausgehen [8]. In Vorversuchen wurde während der Mitverbrennung von 5 Gew.-\% Klärschlamm mit Braunkohle eine erhöhte $\mathrm{PO}_{4}{ }^{3-}$-Konzentration im Rauchgas festgestellt, was die Vorüberlegungen zur Phosphatbildung bestätigte [9].

Mit den im Folgenden beschriebenen experimentellen Untersuchungen wurde die Katalysatordeaktivierung durch gasförmige P-Verbindungen im Labormaßstab sowie in kohlestämmigem Rauchgas nachgewiesen.

\section{Experimentelle Untersuchungen 2.1 Laborversuche}

Der Einfluss des Phosphors wurde in einem Mikroreaktor in synthetischem Rauchgas mit $5 \% \mathrm{O}_{2}, 4,5 \% \mathrm{H}_{2} \mathrm{O}, 600$ ppm NO und $455 \mathrm{ppm} \mathrm{SO}_{2}$ untersucht (s. Abb. 1). Nach einer vierstündigen Konditionierung im Rauchgas wurde handelsübliches Katalysatormaterial jeweils $4 \mathrm{~h} \mathrm{H}_{3} \mathrm{PO}_{4}$-Konzentrationen von 500, 1000 und 1500 ppm in turbulenter Strömung ausgesetzt. 